\title{
Estimation of Risk of Normal-tissue Toxicity Following Gastric Cancer Radiotherapy with Photon- or Scanned Proton-beams
}

\author{
GRACINDA MONDLANE ${ }^{1,2}$, ANA UREBA ${ }^{1}$, MICHAEL GUBANSKI ${ }^{3}$, \\ PEHR A. LIND ${ }^{4,5}$ and ALBERT SIEGBAHN 4,5 \\ ${ }^{1}$ Department of Physics - Medical Radiation Physics, Stockholm University, Stockholm, Sweden; \\ ${ }^{2}$ Department of Physics, Eduardo Mondlane University, Maputo, Mozambique; \\ ${ }^{3}$ Department of Oncology and Pathology, Karolinska University Hospital, Stockholm, Sweden; \\ ${ }^{4}$ Department of Oncology and Pathology, Karolinska Institute, Stockholm, Sweden; \\ ${ }^{5}$ Department of Oncology, Södersjukhuset, Stockholm, Sweden
}

\begin{abstract}
Background/Aim: Gastric cancer (GC) radiotherapy involves irradiation of large tumour volumes located in the proximities of critical structures. The advantageous dose distributions produced by scanned-proton beams could reduce the irradiated volumes of the organs at risk (OARs). However, treatment-induced side-effects may still appear. The aim of this study was to estimate the normal tissue complication probability (NTCP) following proton therapy of GC, compared to photon radiotherapy. Patients and Methods: Eight GC patients, previously treated with volumetric-modulated arc therapy (VMAT), were retrospectively planned with scanned proton beams carried out with the single-field uniform-dose (SFUD) method. A beam-specific planning target volume was used for spot positioning and a clinical target volume (CTV) based robust optimisation was performed considering setup- and rangeuncertainties. The dosimetric and NTCP values obtained with the VMAT and SFUD plans were compared. Results: With SFUD, lower or similar dose-volume values were obtained for OARs, compared to VMAT. NTCP values of $0 \%$ were determined with the VMAT and SFUD plans for all OARs $(p>0.05)$, except for the left kidney $(p<0.05)$, for which lower toxicity was estimated with SFUD. Conclusion: The NTCP reduction, determined for the left kidney with $S F U D$, can be of clinical relevance for preserving renal function after radiotherapy of $G C$.
\end{abstract}

This article is freely accessible online.

Correspondence to: Gracinda Mondlane, Department of Physics, Division of Medical Radiation Physics, Stockholm University, Box 260, 17176 Stockholm, Sweden. Tel: +46 858580000, e-mail: gracinda.mondlane@ki.se

Key Words: NTCP, gastric cancer, VMAT, SFUD, treatment planning.
Gastric cancer (GC) has traditionally been treated with surgery, which remained the sole curative modality for many years. However, most GC patients are diagnosed in late stages, and therefore do not present a good prognosis. Different clinical trials have reported increased overall survival if these patients received post-operative chemoradiotherapy (CRT) (1) or perioperative chemotherapy (2).

GC radiotherapy (RT) is generally being performed with photon beams. The target volumes in these patients are large, with reported sizes of the planning target volume (PTV) in the range between 634 and $1,677 \mathrm{~cm}^{3}$ (3). The PTVs are also located in the immediate proximity of several organs at risk (OARs). Regardless of whether 3D-conformal or arc therapy is utilized, the treatment remains toxic and it is in most cases difficult to preserve the function of both kidneys. Compared to routinely used photon beams, the use of proton beams in RT have been shown to enable a reduction of volumes of healthy tissue irradiated, owing to their dose deposition characteristics. There is also a rising interest in the pencilbeam scanning (PBS) technique in proton beam therapy (PBT), with which a greater flexibility in the dose delivery is made possible. For certain treatments, it may replace the earlier used passive-scattering technique.

Despite the fact that patients have been treated with PBT since more than half a century, its clinical benefit for different tumour sites remains uncertain. Treatment planning comparisons may then be used for estimating the benefits of PBS-based PBT, in relation to photon beam techniques, in for example RT of GC. The dosimetric advantages of using proton beams for GC treatment, in terms of reduction of doses given to the OARs, has already been demonstrated for treatments performed with the passive scattering technique (4) and with PBS using the single-field uniform dose (SFUD) method (5). However, it is also relevant to estimate the advantages of PBS in terms of reduction of the treatmentrelated side effects, to determine if there are any important 
clinical gains in changing the therapeutic method. Predictive models can be employed to estimate the normal tissue complication probability (NTCP) following RT. This study aimed to evaluate the potential to reduce the normal tissue toxicity in the postoperative RT of GC with PBS, compared to photon beam RT performed with volumetric-modulated arc therapy (VMAT).

\section{Patients and Methods}

Patients and structure delineation. Eight consecutive GC patients (median age 60 years, range $=41-68$ years) who were referred to postoperative CRT, were included in this study. All patients had been treated with neoadjuvant chemotherapy followed by total (1 patient) or partial gastrectomy (7 patients). Patients with metastatic disease were excluded. The planning computed tomography (CT) scans were performed 4-7 weeks after the surgery. For all patients, the delineation of structures, performed on the planning CT sets, was based on the pre- and post-operative diagnostic CT images and on the results of the pre-operative gastroscopy. The delineation of the clinical target volume (CTV) was made in accordance with the CRITICS trial (clinicaltrials.gov NCT00407186) protocol and its delineation atlas. The CTVs included the gastric bed/gastric remnant, the anastomoses and lymph node stations. The included lymph nodes depended on the location of the tumour in the stomach in accordance with the Maruyama data $(6,7)$. An isotropic margin of $1.0 \mathrm{~cm}$ was added to the CTV to create the PTV used in the photon beam RT planning. The OARs delineated were the liver, both kidneys, heart, the spinal cord and the part of the bowel excluding the PTV (bowelPTV). The bowel-PTV included both the large and small bowel from the diaphragm down to $1.5 \mathrm{~cm}$ below the PTV.

Treatment planning. The treatment planning for both the photonand proton-beam therapies was performed with the Eclipse treatment planning system (TPS) version 13.7 (Varian Medical Systems, Palo Alto, CA, USA). The photon beam treatment planning was performed with $6 \mathrm{MV}$ photon beams produced by a Varian linear accelerator (Varian Medical Systems). The VMAT technique, delivered with two full arcs, was used for the actual patient treatments. The total prescribed dose was $45 \mathrm{~Gy}$, delivered in daily fractions of $1.8 \mathrm{~Gy}$ (the overall treatment time was 5 weeks). The analytical anisotropic algorithm (AAA) was used for the photon dose calculation and the plans were optimized with the objective of covering the PTV with doses between $95 \%$ and $107 \%$ of the prescribed dose. Regarding the normal tissue constraints, the mean liver dose was not allowed to exceed $30 \mathrm{~Gy}$ and the permitted maximum dose given to the spinal cord was set to 45 Gy. In every treatment plan, efforts were made to spare one of the kidneys as much as possible. The maximum dose allowed to $2 / 3$ of this kidney should not exceed $40 \%$ of the prescribed dose. Attempts were also made to minimise irradiation to the heart and bowel-PTV.

In the treatment planning performed with proton beams, PBS implemented with the SFUD method was used. A two-field configuration with one frontal and one lateral field (incident from the left) was chosen. The beam angles were adapted for each patient with the aim of avoiding unnecessary irradiations of the OARs. Spotscanned proton beams with kinetic energies between 60 and 230 $\mathrm{MeV}$, produced by an IBA machine (Ion Beam Applications, S.A., Louvain-La-Neuve, Belgium), were used for the PBT planning. A range shifter of water equivalent thickness of $3.5 \mathrm{~g} / \mathrm{cm}^{2}$ was used in the two proton fields to enable full dose-coverage in the proximal part of the target volume. The proton dose calculation was done using the proton convolution superposition algorithm (PSC) and the robust optimization was performed with the Nonlinear Universal Proton Optimizer (NUPO) algorithm. To ensure satisfactory targetdose coverage, beam-specific PTVs (bsPTV) were created for each beam, using a functionality available in Eclipse TPS, as described by Park et al. (8). This approach takes into account setup errors, range uncertainties and heterogeneities along the beam path, to design a PTV for each individual field. The dose calculation and CTV-based robust optimization were done on CT sets in which the air cavities along the beam path and within the target volume had been replaced with water equivalent material. An isotropic setup uncertainty of $1.0 \mathrm{~cm}$ and a proton range uncertainty of $3.5 \%$ were considered during the robust optimisation, which resulted in 12 different scenarios with perturbed dose distributions and one nominal dose distribution (without perturbations). The plans created in this manner were called the SFUD ${ }_{\text {opt }}$ plans. Further, to evaluate the plan robustness against possible density changes, the $\mathrm{SFUD}_{\text {opt }}$ plans were recalculated based on the original $\mathrm{CT}$ sets containing air cavities, to produce the $\mathrm{SFUD}_{\mathrm{ver}}$ plans. The planning constraints set for the OARs in the VMAT planning were used also in the PBT planning. The robustness criterion for the CTV was set to $V_{95}>98 \%$ for at least 10 out of the 12 simulated scenarios. A generic relative biologic effectiveness (RBE) of 1.1 was assumed for the proton beams.

Dosimetric evaluation of the treatment plans. A robustness analysis

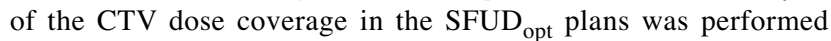
based on the robustness criterion. For the VMAT plans, the nominal $\mathrm{SFUD}_{\text {opt }}$ plans and the $\mathrm{SFUD}_{\mathrm{ver}}$ plans, values of $\mathrm{D}_{2}, \mathrm{D}_{98}, \mathrm{~V}_{95}$ and the homogeneity index (HI) [(D2 - D98)/D prescribed $]$ were registered for all patients. The dose-volume values for the OARs specified in the QUANTEC recommendations were also registered (mean dose and $\mathrm{V}_{18}$ for each kidney, $\mathrm{V}_{20}$ for the combined kidneys, $\mathrm{D}_{\max }$ for the spinal cord, mean dose and $\mathrm{V}_{20}$ for the liver, $\mathrm{V}_{25}$ for the heart and $\mathrm{V}_{45}$ for the bowel-PTV).

Estimation of NTCP. The NTCP was estimated using the LymanKutcher-Burman (LKB) model $(9,10)$. The LKB model is applicable under conditions of homogeneous irradiation of the OARs. In order to accommodate the clinical data, which have been obtained for inhomogeneous irradiations, the non-uniform dose-volume histograms (DVHs) were transformed into equivalent DVHs for uniform irradiations of the OARs with the maximum dose in the DVHs (11). In addition, the DVHs were converted to equivalent 2-Gy per-fraction treatment DVHs. The NTCP-model parameters used in the calculations and the corresponding endpoints are shown in Table I.

The dosimetric and NTCP values obtained with the two SFUD plans were compared pairwise with the values determined with the VMAT plans using a Wilcoxon two-sided statistical test with a significance level of 0.05 .

\section{Results}

Dosimetric evaluation. For all patients, the robustness criterion specified for the CTV was fulfilled with the SFUD $_{\text {opt }}$ plans. The planning objectives set for the PTV in the VMAT plans were also reached. The constraints set for the OARs in the VMAT- and SFUD-plans were respected for 

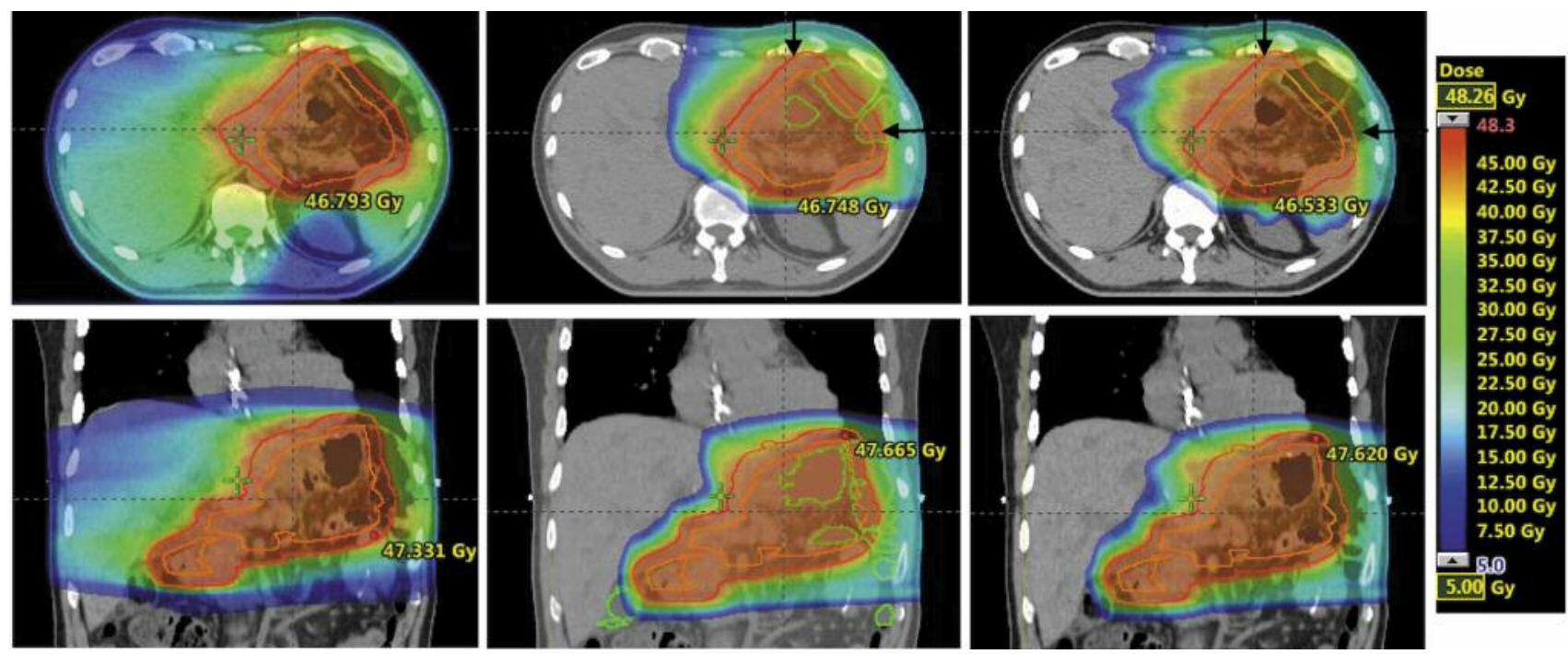

Figure 1. Dose distributions obtained in the axial (top) and frontal (bottom) planes for patient \#5 with the VMAT (A), SFUD ${ }_{\text {opt }}(B)$ and $S F U D_{\text {ver }}$ (C) plans. Doses are presented in Gy (IsoE) and the arrows indicate the direction of the proton beam incidence.

Table I. Lyman-Kutcher-Burman (LKB) model parameters for the organs at risk (OARs).

\begin{tabular}{|c|c|c|c|c|c|c|}
\hline OAR & $\mathrm{n}$ & $\mathrm{m}$ & TD50 (Gy) & Endpoint & $\alpha / \beta$ (Gy) & Source \\
\hline Kidney & 0.7 & 0.1 & 28 & Clinical nephritis & $3^{\mathrm{a}}$ & Burman et al. (1991) (9) \\
\hline Spinal cord & 0.05 & 0.175 & 66.5 & Radiation myelitis & $0.87^{\mathrm{b}}$ & Burman et al. (1991) (9) \\
\hline Liver & 0.97 & 0.12 & 45.8 & Radiation induced liver disease (RILD) & 2 & Dawson et al. (2002) (21) \\
\hline Heart & 0.636 & 0.13 & 50.6 & Pericarditis & $3^{\mathrm{b}}$ & Martel et al. (1998) (22) \\
\hline Bowel & 0.17 & 0.11 & 55 & Obstruction/Perforation & $3^{\mathrm{a}}$ & Burman et al. (1991) (9) \\
\hline
\end{tabular}

aAssumed for these OARs; ${ }^{b} \alpha / \beta$ value for the heart was taken from Gagliardi et al. (1996) (23) and for spinal cord was taken from Schultheiss (2008) (24).

all patients. The dose distributions calculated with the

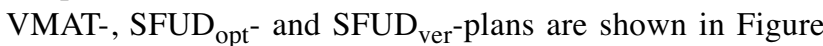
1 for one representative patient case (patient \#5). The median DVHs obtained for the CTVs and the OARs with the VMATand the two SFUD-plans are shown in Figure 2.

A median HI value of 0.07 (range=0.05-0.11 HI) was calculated for the CTV with the VMAT plans while median $\mathrm{HI}$ values of 0.02 (range $=0.01-0.05 \mathrm{HI}$ ) and 0.05 (range $=0.02$ $0.09 \mathrm{HI}$ ) were obtained with the $\mathrm{SFUD}_{\mathrm{opt}^{-}}$and $\mathrm{SFUD}_{\mathrm{ver}}$-plans, respectively. In terms of dose coverage of the CTV, a median value of V95 of $100 \%$ was determined for the three plans. However, for one patient a V95 of $95 \%$ was registered with the SFUDver plan (Table II). Regarding the OARs, both the $\mathrm{SFUD}_{\mathrm{opt}^{-}}$and $\mathrm{SFUD}_{\text {ver }}$-plans provided a decrease in the dosevolume values determined for the kidneys, spinal cord and liver, $p<0.05$ (Table II). However, for the heart and bowelPTV, no significant differences in the dosimetric values were obtained with the VMAT and the two SFUD plans $(p>0.05)$.
For all the delineated structures studied, there was no significant difference between the dosimetric values obtained with the nominal SFUD $_{\text {opt }}$ plans and the recalculated SFUD $_{\text {ver }}$ plans (Table II), which indicated that robustness against the introduced density changes was achieved.

NTCP evaluation. The calculated NTCPs for the right kidney, spinal cord, liver, heart and bowel-PTV was $0 \%$ for all patients with the VMAT-, SFUD opt $^{-}$and SFUD $_{\text {ver }^{-}}$ plans. On the other hand, for the left kidney, an NTCPvalue of $0 \%$ was determined for 3 patients with the VMAT plans, while for the remaining 5 patients, NTCPs between $7 \%$ and $86 \%$ were obtained. With the SFUD opt $^{- \text {plans, an }}$ NTCP of $0 \%$ was obtained for the left kidney for all

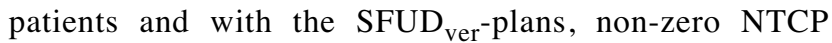
values (14\% and $20 \%$ ) were determined for only 2 patients. A summary of the calculated NTCP values for the left kidney is shown in Figure 3. 

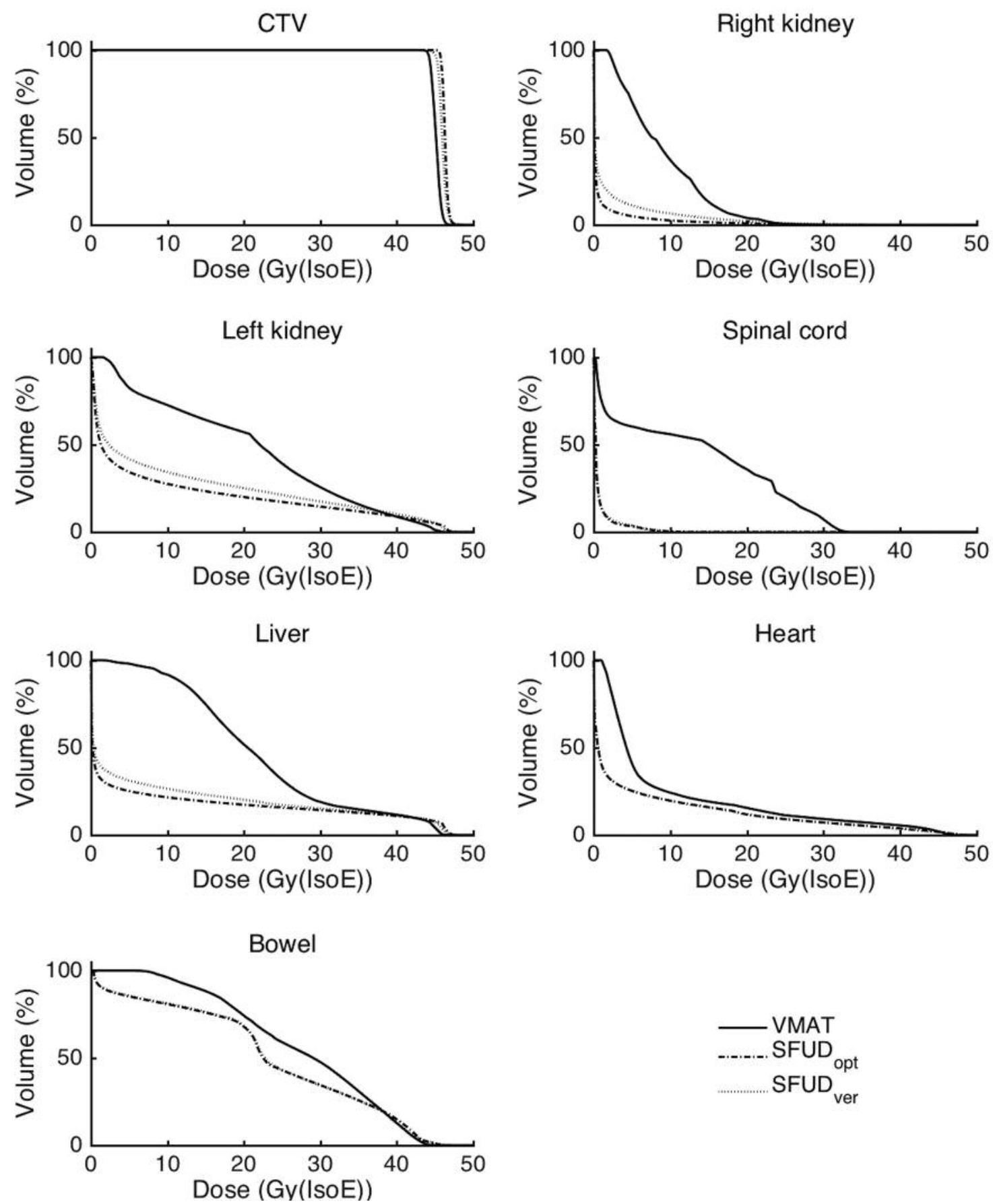

Figure 2. Median dose-volume histograms (DVHs) determined for the organs at risk (OARs), for all patients, with the VMAT (full lines), SFUD opt (dot-dashed lines) and $S F U D_{\text {ver }}$ (dotted lines) plans.

\section{Discussion}

Normal tissue toxicity following photon beam CRT of GC has been reported in an early clinical trial conducted by Macdonald et al. (2). In this trial (2), $17 \%$ of the patients included could not continue with the treatment due to the toxicities that were observed. It is, however, relevant to stress that some of the sequelae observed were not exclusively RT-related since the patients received chemotherapy as well. The significantly reduced NTCP 
Table II. Median values (range) of the dosimetric values obtained for the clinical target volumes (CTV) and the organs at risk (OARs) with the volumetric-modulated arc therapy (VMAT) and single-field uniform dose (SFUD) plans.

\begin{tabular}{lccc}
\hline Structure & VMAT & SFUD $_{\text {opt }}$ & SFUD $_{\text {ver }}$ \\
\hline CTV & & & \\
$\mathrm{HI}$ & $0.07(0.05-0.11)$ & $0.02(0.01-0.05)$ & $0.05(0.02-0.09)$ \\
$\mathrm{V}_{95}(\%)$ & $100(100-100)$ & $100(100-100)$ & $100(95-100)$ \\
R Kidney & & & \\
$\mathrm{D}_{\text {mean }}(\mathrm{GyIsoE})$ & $10.1(3.2-15.5)$ & $1.6(0.0-2.5)$ & $2.0(0.1-3.1)$ \\
$\mathrm{V}_{18}(\%)$ & $7.1(0-22.8)$ & $0.7(0-4.4)$ & $2.8(0-5.6)$ \\
L Kidney & & & \\
$\mathrm{D}_{\text {mean }}(\mathrm{GyIsoE})$ & $24.1(9.3-32.0)$ & $9.6(0.4-17.3)$ & $11.4(2.4-24.4)$ \\
$\mathrm{V}_{18}(\%)$ & $66.0(11.2-100)$ & $21.2(3.0-40.0)$ & $26.5(4.0-63.3)$ \\
Combined Kidneys & & & \\
$\mathrm{V}_{20}(\%)$ & $33.5(14.5-56.7)$ & $10.2(1.3-23.4)$ & $12.8(2.6-36.5)$ \\
Spinal cord & & & \\
$\mathrm{D}_{\text {max }}(\mathrm{GyIsoE})$ & $34.1(25.9-37.3)$ & $9.2(0.5-22.8)$ & $9.0(0.6-22.8)$ \\
Liver & & & \\
$\mathrm{D}_{\text {mean }}(\mathrm{GyIsoE})$ & $23.6(19.4-25.6)$ & $8.2(6.8-12.9)$ & $9.2(8.2-13.9)$ \\
$\mathrm{V}_{20}(\%)$ & $52.9(30.0-61.7)$ & $17.4(15.0-29.0)$ & $20.1(17.5-31.0)$ \\
Heart & & & \\
$\mathrm{V}_{25}(\%)$ & $11.3(3.1-22.7)$ & $9.1(3.2-27.0)^{*}$ & $9.2(3.5-27.0)^{*}$ \\
Bowel-PTV & & & \\
$\mathrm{V}_{45}\left(\mathrm{~cm}{ }^{3}\right)$ & $2.5(0.0-87.6)$ & $3.3(0.0-47.9)^{*}$ & $1.5(0.0-16.7)^{*}$ \\
\hline
\end{tabular}

${ }^{*} p>0.05$, compared to the values obtained with VMAT.

values calculated in our study for the left kidney with the SFUD plans could be of clinical relevance to preserve renal function and to make treatment feasible for patients with already reduced kidney function. Similar findings were obtained in studies of sarcoma and pancreatic cancer patients for whom proton treatments were reported to provide an improved sparing of the OARs in the urinary tract $(12,13)$. The importance of sparing the kidneys in RT-treated GC patients has also been discussed in a study involving $87 \mathrm{GC}$ patients, conducted by Trip et al. (14), in which the twodimensional photon-planning technique was compared with three-dimensional conformal RT and intensity-modulated radiation therapy (IMRT). The authors found that a significantly lower dose was given to the left kidney with IMRT. This dose reduction was also found to be of clinical importance, as the kidney function was reported to decrease at a lower rate in the IMRT treated patients.

In terms of the dosimetric evaluation carried out in this study for the OARS, an improved sparing of the kidneys, spinal cord and liver was achieved with the SFUD method. However, this did not lead to reductions in the estimated NTCPs for the spinal cord and the liver, as NTCP values of $0 \%$ were obtained with both the VMAT- and the SFUDplans. The reason that low toxicity levels were calculated in this study was related to the low fraction doses given to the patients and the overall long treatment time. The GC RT

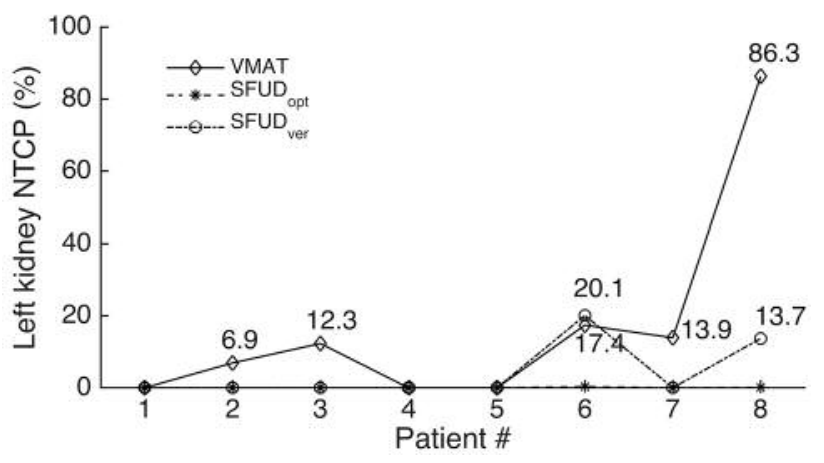

Figure 3. NTCPs calculated for the left kidney with the VMAT, SFUD and $S F U D_{\text {ver }}$ plans, for all patients.

treatments are delivered in combination with chemotherapy and in a post-operative setting. Therefore, the prescribed fraction doses are low (1.8 Gy(IsoE)). This indicates the importance of using not only the dosimetric parameters in the plan comparison, but also the results from toxicity predictions. As suggested by Langendijk et al. (15), NTCP predictions can also be used in treatment-plan evaluations for selecting patients for which PBT could provide significant NTCP reductions. In our study, some patients would be indicated for PBT, based on the kidney sparing possibilities.

The use of PBS in the treatment of tumour sites influenced by density changes, for example GC, requires that approaches that guarantee plan robustness should be implemented. In our study, we used the density override approach to replace the air cavities in the CT sets used for dose calculation with water equivalent HUs. This resulted in increased doses given to the OARs located beyond the distal

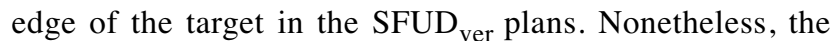
dosimetric changes in the SFUD ${ }_{\text {ver }}$ plans, compared to the SFUD $_{\text {opt }}$ plans, were not significant for the patients included in this study. This showed that robustness against density changes was achieved. In a study of PBT of GC conducted by Dionisi et al. (4), the density override approach was shown to minimise the proton range uncertainties due to bowel gas movement. Furthermore, beam angles can be selected to guarantee that the dose distributions are minimally affected by the inter-fraction density changes along the beam path. Previous studies, exploring the role of PBT for treatments in the upper abdominal region, focused on patients with pancreatic cancer, seminoma, sarcoma and oesophageal cancer. Typically, a field-setup with a posterior field in combination with a field entering from the right, passing through the liver and/or an anterior beam have been used in these studies in order to avoid the density heterogeneities related to intestinal gas variations $(12,13,18$, 19). In the SFUD plans prepared for this study, a frontal and 
a lateral field incident from the left of the patient was chosen. This setup was chosen to avoid irradiation through the liver and hence to improve the sparing of the liver.

The NTCP assessment performed in this study was made under the assumption that the NTCP-model parameters, obtained from patient follow-up studies after photon RT, were also valid for proton-beam treatments. This assumption is commonly made in comparative radiotherapy studies due to the lack of biological-model parameters obtained for proton beam treatments. However, the increased clinical use of proton therapy will provide more reliable values of these model-parameters for the different OARs. Moreover, it is also important to consider other sources of uncertainties in the assessment of risks of normal tissue toxicity following RT. These include, among others, the use of different algorithms for dose-calculation, which influences the produced dose distributions, and hence, both the dosimetric parameters used to determine the tolerance values for different toxicities (16) and the estimated NTCP values (17).

In previous reports of PBT of GC, the photon-PTV was used as the target volume also in the PBT planning $(4,5)$. This approach does not account for the proton-specific uncertainties (20). In the present study, CTV-based bsPTV (8) was used to achieve a satisfactory CTV dose coverage. A CTV-based robust optimisation, which involved isocenter shifts and changes in the range of the proton beams, was also performed. This resulted in plans which were robust in the presence of density changes, as was observed in this study

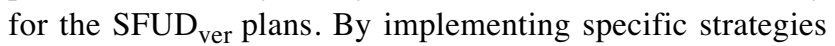
for addressing the different uncertainties in PBT, plans which fulfil the robustness criteria for both the target volume and the OARs can be created.

\section{Conclusion}

The NTCPs calculated with the proton SFUD method were similar to those calculated with the photon VMAT technique for all the OARs studied, except for the left kidney. The significant reduction of the NTCP estimated for the left kidneys with the proton plans could be of clinical relevance for preserving renal function after RT of GC. The use of robust optimisation in the proton therapy planning for GC could contribute to improvements of the plan quality, both in terms of target coverage and normal-tissue sparing.

\section{Acknowledgements}

This study was financially supported by the Swedish International Development Cooperation Agency (SIDA) through the International Science Programme (ISP) and the Cancer Research Funds of Radiumhemmet at Karolinska Institute, Stockholm, Sweden. The Authors would also like to acknowledge Bruno Sorcini for the discussions and information, which contributed to improve the work presented in this article.

\section{Conflicts of Interest}

The Authors have no conflicts of interest to disclose.

\section{References}

1 Cunningham D, Allum WH, Stenning SP, Thompson JN, Van de Velde CJH, Nicolson M, Scarffe JH, Lofts FJ, Falk SJ, Iveson TJ, Smith DB, Langley RE, Verma M, Weeden S and Chua YJ: Perioperative chemotherapy versus surgery alone for resectable gastroesophageal cancer. N Engl J Med 355: 11-20, 2006.

2 Macdonald JS, Smalley SR, Benedetti J, Hundahl SA, Estes NC, Stemmermann GN, Haller DG, Ajani JA, Gunderson LL, Jessup JM and Martenson JA: Chemoradiotherapy after surgery compared with surgery alone for adenocarcinoma of the stomach or gastroesophageal junction. N Engl J Med 345: 725730, 2001.

3 Jansen EPM, Nijkamp J, Gubanski M, Lind PARM and Verheij $\mathrm{M}$ : Interobserver variation of clinical target volume delineation in gastric cancer. Int J Radiat Oncol Biol Phys 77: 1166-1170, 2010.

4 Dionisi F, Amichetti M, Avery S, Lukens JN, Ding X, Kralik J, Kirk M, Metz JM, Plastaras JP and Roses RE: Proton therapy in adjuvant treatment of gastric cancer: Planning comparison with advanced X-ray therapy and feasibility report. Acta Oncol (Madr) 53: 1312-1320, 2014

5 Mondlane G, Gubanski M, Lind PA, Ureba A and Siegbahn A: Comparison of gastric-cancer radiotherapy performed with volumetric modulated arc therapy or single-field uniform-dose proton therapy. Acta Oncol (Madr) 56: 832-838, 2017.

6 Maruyama K, Gunvén P, Okabayashi K, Sasako M and Kinoshita T: Lymph node metastases of gastric cancer: General pattern in 1931 patients. Ann Surg 210: 596-602, 1989.

7 Tepper J and Gunderson LL: Radiation treatment parameters in the adjuvant postoperative therapy of gastric cancer. Semin Radiat Oncol 12: 187-195, 2002.

8 Park PC, Zhu XR, Lee AK, Sahoo N, Melancon AD, Zhang L and Dong L: A Beam-Specific Planning Target Volume (PTV) design for proton therapy to account for setup and range uncertainties. Int J Radiat Oncol 82: e329-e336, 2012.

9 Burman C, Kutcher GJ, Emami B and Goitein M: Fitting of normal tissue tolerance data to an analytic function. Int J Radiat Oncol Biol Phys 21: 123-135, 1991.

10 Lyman JT: Complication probability as assessed from dosevolume histograms. Radiat Res 104: S13, 1985.

11 Kutcher GJ and Burman C: Calculation of complication probability factors for non-uniform normal tissue irradiation: The effective volume method. Int J Radiat Oncol 16: 1623-1630, 1989.

12 Nichols RC, Huh SN, Prado KL, Yi BY, Sharma NK, Ho MW, Hoppe BS, Mendenhall NP, Li Z and Regine WF: Protons offer reduced normal-tissue exposure for patients receiving postoperative radiotherapy for resected pancreatic head cancer. Int J Radiat Oncol Biol Phys 83: 158-163, 2012.

13 Swanson EL, Indelicato DJ, Louis D, Flampouri S, Li Z, Morris CG, Paryani N and Slopsema R: Comparison of threedimensional (3D) conformal proton radiotherapy (RT), 3D conformal photon RT, and intensity-modulated RT for retroperitoneal and intra-abdominal sarcomas. Int J Radiat Oncol Biol Phys 83: 1549-1557, 2012. 
14 Trip AK, Nijkamp J, Van Tinteren H, Cats A, Boot H, Jansen EPM and Verheij M: IMRT limits nephrotoxicity after chemoradiotherapy for gastric cancer. Radiother Oncol 114: 421-426, 2015.

15 Langendijk JA, Lambin P, De Ruysscher D, Widder J, Bos M and Verheij M: Selection of patients for radiotherapy with protons aiming at reduction of side effects: The model-based approach. Radiother Oncol 107: 267-273, 2013.

16 Flejmer AM, Dohlmar F, Nilsson M, Stenmarker M and Dasu A: Analytical anisotropic algorithm versus pencil beam convolution for treatment planning of breast cancer: implications for target coverage and radiation burden of normal tissue. Anticancer Res 35: 2841-2848, 2015

17 Hedin E and Bäck A: Influence of different dose calculation algorithms on the estimate of NTCP for lung complications. J Appl Clin Med Phys 14: 127-139, 2013.

18 Hoppe Bradford S, Mamalui-Hunter Maria P, Mendenhall Nancy $\mathrm{J}$, Li Zuofeng $\mathrm{J}$ and Indelicato Daniel $\mathrm{J}$ : Improving the therapeutic ratio by using proton therapy in patients with stage I or II seminoma. Am J Clin Oncol 36: 31-37, 2013.

19 Zhang X, Zhao K-L, Guerrero TM, Mcguire SE, Yaremko B, Komaki R, Cox JD, Hui Z, Li Y, Newhauser WD, Mohan R and Liao Z: Four-dimensional computed tomography-based treatment planning for intensity-modulated radiation therapy and proton therapy for distal esophageal cancer. Int J Radiat Oncol Biol Phys 72: 278-287, 2008.
20 Engelsman M and Bert C: Precision and uncertainties in Proton Therapy of moving targets. In: Proton Therapy Physics. Paganetti H (ed.). CRC Press, pp. 435-460, 2012.

21 Dawson LA, Normolle D, Balter JM, McGinn CJ, Lawrence TS and Ten Haken RK: Analysis of radiation-induced liver disease using the Lyman NTCP model. Int J Radiat Oncol 53: 810-821, 2002.

22 Kaye Martel M, Sahijdak WM, Ten Haken RK, Kessler ML and Turrisi AT: Fraction size and dose parameters related to the incidence of pericardial effusions. Int J Radiat Oncol Biol Phys 40: 155-161, 1998

23 Gagliardi G, Lax I, Ottolenghi A and Rutqvist LE: Long-term cardiac mortality after radiotherapy of breast cancer - application of the relative seriality model. Br J Radiol 69: 839-846, 1996.

24 Schultheiss TE: The Radiation dose-response of the human spinal cord. Int J Radiat Oncol 71: 1455-1459, 2008.
Received March 2, 2018

Revised March 25, 2018

Accepted March 26, 2018 